\title{
CONF-9505219--2
}

Note: This is a preprint of a paper being submitted for publication. Contents of this paper should not be quoted nor referred to without permission of the author(s).

Third International Conference on Laser Ablation (COLA'95)

Strasbourg, France

\section{LASER ABLATION PLUME THERMALIZATION DYNAMICS IN \\ BACKGROUND GASES: COMBINED IMAGING, OPTICAL ABSORPTION AND EMISSION SPECTROSCOPY, AND ION PROBE MEASUREMENTS}

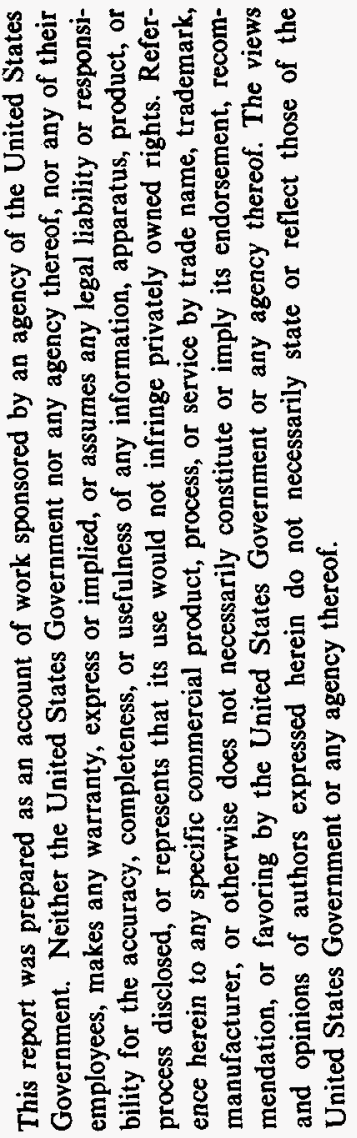

David B. Geohegan and A. A. Puretzky*

Solid State Division

Oak Ridge National Laboratory

*Institute of Spectroscopy

Russian Academy of Sciences, Troitsk, Russia

February 1995

\footnotetext{
"The submitted manuscript has been authored by a contractor of the U.S. Government under

contract No. DE-AC05-840R21400.

Accordingly, the U.S. Government retains a

nonexclusive, royalty-free license to publish

or reproduce the published form of this

contribution, or allow others to do so, for

U.S. Government purposes."
}

\section{Prepared by}

Solid State Division

Oak Ridge National Laboratory

P. O. Box 2008

Oak Ridge, Tennessee 37831-6056

managed by

LOCKHEED MARTIN ENERGY SYSTEMS, INC.

for the

U.S. DEPARTMENT OF ENERGY

under contract DE-AC05-84OR21400

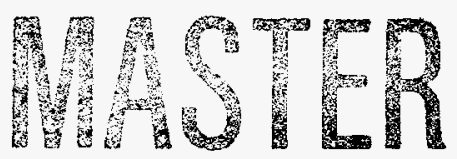




\section{DISCLAIMER}

Portions of this document may be illegible in electronic image products. Images are produced from the best available original document. 
Laser Ablation Plume Thermalization Dynamics in Background Gases: Combined Imaging, Optical Absorption and Emission Spectroscopy, and Ion Probe Measurements

David B. Geohegan and Alexander A. Puretzky*

Solid State Division, Oak Ridge National Laboratory, Oak Ridge, Tennessee 37831-6056.

*Institute of Spectroscopy, Russian Academy of Sciences, Troitsk, Russia

\section{ABSTRACT}

Combined diagnostic measurements are employed to characterize the penetration of energetic ablation plumes through background gases during a key transitional regime in which the ion flux is observed (with fast ion probes) to split into distinct fast and slowed components. This apparently general phenomenon occurs over a limited range of distances at ambient pressures typically used for PLD (as reported for YBCO ablation into $\left.\mathrm{O}_{2}\right)^{1-5}$ and may be important to film growth by PLD because a 'fast' component of ions can arrive at the probe (or substrate) with little or no delay compared to propagation in vacuum (i.e., high 10-100 eV kinetic energies). At longer distances, this 'fast' component is completely attenuated, and only slowed distributions of ions are observed. Interestingly, this 'fast' component is easily overlooked in imaging studies because the bright plume luminescence occurs in the slowed distribution.

Time- and spatially-resolved optical absorption and emission spectroscopy are applied to experimentally determine the composition of the 'fast' and 'slow' propagating plume components for a single-component target ablation (yttrium) into an inert gas (argon) for correlation with quantitative imaging and ion probe measurements. The yttrium/argon system was chosen because optical absorption spectroscopy of both $\mathrm{Y}$ and $\mathrm{Y}^{+}$was simultaneously possible ${ }^{9}$ and the inert nature of argon. Experimental results for several other systems, including $\mathrm{Si} / \mathrm{Ar}, \mathrm{Si} / \mathrm{He}, \mathrm{YBCO} / \mathrm{O}_{2}$ are presented to illustrate variations in scattering mechanisms. Speciesresolved imaging of $\mathrm{YO}^{*}$ and $\mathrm{Ba}^{*}$ is presented for the $\mathrm{YBCO} / \mathrm{O}_{2}$ system to illustrate the similarities and differences in the spatial regions of observed luminescence. These measurements confirm that, in addition to the bright significantly-slowed front which has been 
described by shock or drag propagation models, ${ }^{1}$ a fast-component of target material is transmitted to extended distances for some ambient pressures with near-initial velocities.

\section{INTRODUCTION}

The ability of energetic ablation plasma plumes to penetrate low-pressure background gases during PLD is a key processing advantage. However, fundamental details of how the plasma plume, or 'beam,' of target material is scattered, slowed, and attenuated by background gas collisions are still being revealed. Key processing parameters of kinetic energy, density, temperature, and fractional ionization can undergo radical changes at a given deposition-distance for small changes in background gas pressure as effects initiated by the background gas collisions, such as shock-heating or clustering, can significantly alter the beam properties.

One such effect is investigated here. Reported here are combined diagnostic measurements during a key transitional regime in which the ion flux is observed to split into distinct fast and slowed components. This apparently general phenomenon occurs over a limited range of distances at ambient pressures, including those sometimes used for PLD (as reported for YBCO ablation into $\mathrm{O}_{2}$ ). 1,2,6,7 This "plume-splitting" is significant because a 'fast' component of ions can arrive at the ion probe (or substrate) with little or no delay compared to propagation in vacuum (e.g., $\mathrm{Y}^{+}$kinetic energies in this study were up to $250 \mathrm{eV}$, and $\mathrm{Si}^{+}$up to $215 \mathrm{eV}$ ). Kools recently employed a Monte Carlo simulation involving elastic collisions to prove this possibility. ${ }^{8}$ However, at longer distances this 'fast' component is completely attenuated, and a single, slowed distribution of ions is observed. This 'fast' component is easily overlooked in imaging studies because enhanced plume luminescence occurs in the slowed distribution. However, the transmitted flux is readily observed with fast ion probes which provide convenient measurements of the magnitude and time-of-flight of the ion current (flux) in plasma plumes propagating through background gases. 1,2,5-7,9

In this paper, time- and spatially-resolved optical absorption and emission spectroscopy are applied to determine the composition of the 'fast' and 'slow' propagating plume components for single-component target ablation (yttrium) into an inert gas (argon). These measurements are correlated with quantitative imaging and ion probe measurements. Although the splitting effect

$$
\text { F-II.8 Geohegan, et.al. p. } 2
$$


appears quite general, the yttrium/argon system was chosen because optical absorption spectroscopy of both $\mathrm{Y}$ and $\mathrm{Y}^{+}$was simultaneously possible 10 and because argon is chemically inert.

\section{EXPERIMENTAL}

The experimental apparatus has been described previously. ${ }^{4-7,10}$ A cylindrical lens $\left(\mathrm{f}_{\mathrm{L}}=\right.$ $500 \mathrm{~mm}$ ) focused the apertured 248-nm beam from a Questek $2960 \mathrm{KrF}$-excimer laser (25 $\mathrm{ns}$ FWHM pulse width) to a horizontal line $(2.0 \mathrm{~cm} \times 0.075 \mathrm{~cm})$ on the face of a sanded yttrium (99.99\%) pellet at an incidence angle of $30^{\circ}$. For optical absorption spectroscopy, a pulsed (1.5 $\mu$ S FWHM) Xe lamp beam (width $0.5 \mathrm{~mm}$ ) was passed parallel to the target surface and just above a wire ion probe tip (floating bias $-70 \mathrm{~V}$ with respect to its shield). The expansion of the ablated yttrium formed a vertical fan which expanded little along the horizontal lamp beam axis (toward the camera or spectrometer), so the absorption path length remained at approximately 2 $\mathrm{cm}$ for the two distances investigated here $(\mathrm{d}=1.5,2.5 \mathrm{~cm})$. Optical detection utilized a 1.33meter spectrometer (McPherson 209, $1800 \mathrm{~g} / \mathrm{mm}$ holographic grating) outfitted with an intensified, gated diode array (Princeton Instruments IRY-700RB, 5-ns resolution) and photomultiplier tube (Hamamatsu R955). Fast unfiltered imaging was performed with a gated, (ICCD), lens-coupled camera system (Princeton Instruments) with variable gain, 200-820 $\mathrm{nm}$ response, and variable gating above 5-ns. Species resolved imaging was performed with a Varispec ${ }^{\mathrm{TM}}$ tunable liquid crystal filter (Cambridge Research Instrumentation, Inc.) with 400-720 $\mathrm{nm}$ range, and a $10 \mathrm{~nm}$ bandwidth.

\section{RESULTS}

Figure 1 serves to summarize the general nature of the ion flux splitting effect noted with ion probes. In Fig. 1(a), the ion probe current measured at $\mathrm{d}=5 \mathrm{~cm}$ following KrF-laser ablation into vacuum and $75 \mathrm{mT}$ Torr oxygen is shown. Under these conditions, two distinct components to the ion flux are evident: a 'fast' component traversing to $\mathrm{d}=5 \mathrm{~cm}$ with little delay compared to vacuum, and a 'slow' component which is significantly slowed. While overall, the ion flux decreases exponentially with distance (or pressure), the 'fast' component becomes completely 
attenuated after a certain distance (or pressure) and the second, 'slow' component is the component which is characterized to propagate in accordance with 'drag' or 'shock' propagation models.

For most pressure-distance combinations, these two components are not easily resolvable. In general, such a distinct separation occurs over a very limited range of pressures at a given distance, if at all. For example, for the ablation of silicon into helium at $d=5 \mathrm{~cm}$ in Fig. 1(b), the pressure must be increased past $150 \mathrm{mTorr}$ before these two components become easily resolvable [as in Fig. 1 (c)]. Figure 1(c) illustrates, in addition, the splitting of the ion flux for the Si/Ar system. In this case, the 'fast' component retains a nearly identical time-of-flight profile with the starting, vacuum pulse. A detailed description of the $\mathrm{Si} / \mathrm{He}$ and $\mathrm{Si} / \mathrm{Ar}$ systems, along with computer simulations of these effects, will be presented elsewhere. As in the case of YBCO penetrating through $\mathrm{O}_{2}, 1,2,6,7$ the ion flux in these $\mathrm{Si} / \mathrm{He}$ and $\mathrm{Si} / \mathrm{Ar}$ studies was attenuated exponentially with background pressure and/or distance in general agreement with the simple scattering model (effective cross sections in both cases $\sim 1 \times 10^{-16} \mathrm{~cm}^{2}$ ) utilized for YBCO. 1,2,6,7

Figure 1(d) presents the splitting of the ion flux at $\mathrm{d}=1.5 \mathrm{~cm}$ following $\mathrm{KrF}$-laser ablation of yttrium into $200 \mathrm{mTorr}$ argon. At this distance and pressure, the diminishing 'fast' component of the ion flux is still an appreciable fraction of the total signal, and is temporally well-resolved from the 'slow' component. In all of the cases shown in Fig. 1, the ion probe indicates material penetrating the background gas with near-vacuum velocities, well ahead of the slowing 'shock' front.

At $1.0 \mu \mathrm{s}$, for example, Fig. 1(d) indicates that the ion flux is nearly peaked in vacuum, and in the midst of the 'fast' component flux in 200 mTorr argon. Corresponding gated images of the visible yttrium-plume luminescence at $\Delta \mathrm{t}=1.0 \mu \mathrm{s}$ in vacuum and $200 \mathrm{mTorr}$ argon are shown in Figs. 2(a) and 2(b). The free expansion in vacuum [Fig. 2(a)] shows luminescence extending to $2.0 \mathrm{~cm}$, while the luminescence in argon [Fig. 2(b)] appears not to reach the ion probe at $\mathrm{d}=1.5 \mathrm{~cm}$. However, this is a result of the scaling of the intensity data to accommodate the bright shock structure [over $10 \times$ brighter than the peak in Fig. 2(a)]. When normalized to the same peak intensity, and replotted in Fig. 2(d) and 2(e) with a 10-grayscale palette, the same two images reveal luminescence extending well past the ion probe to nearly the same distances as in 
vacuum. A line profile along the normal to the irradiated spot (Fig. 2(f)) correctly portrays the relative intensities.

The imaging and ion probe data suggest that a portion of the original target material is transmitted to long distances without significant scattering interactions with the background gas, thereby maintaining velocities nearly equal to those for vacuum propagation. However, it is necessary to prove that this 'fast' component contains fast plume ions (and not, for example, only background gas ions which have somehow been swept-up, or 'snowplowed' in front of the target material).

In order to investigate the composition of the yttrium plume during the splitting of the ion flux, optical absorption spectra were obtained at $\mathrm{d}=1.5 \mathrm{~cm}$ at three times $(1.3 \mu \mathrm{s}, 2.1 \mu \mathrm{s}$, and $2.9 \mu \mathrm{s})$ corresponding to times during the first peak, shoulder, and second peak of the $200 \mathrm{~m}$ Torr ion flux in Fig. 1(d). This spectral region for absorption was chosen because both ground-state neutral $(\mathrm{Y})$ and ground-state ionic $\left(\mathrm{Y}^{+}\right)$populations could simultaneously be investigated (ionic lines indicated by + and neutral lines indicated by $o$ ). In addition, quantitative imaging was performed at the same three times and these images are again plotted in two representations (see figure caption) in Fig. 3(b)-3(d) and Fig. 3(e)-3(g).

At $1.3 \mu$ s [Fig. 3(b) and 3(e)], very weak optical emission is present at the probe position during the first peak of the ion flux signal. However, the optical absorption spectrum [Fig. 3(h)] reveals strong $\mathrm{Y}^{+}$absorption [lines indicated by + in Figs. 3(a)-3(c)] at this time, confirming the penetration of target ions through the background gas at 'vacuum' velocities. At $2.1 \mu \mathrm{s}$ [Fig. 3(c) and 3(f)] the steep gradient of plume luminescence has reached the probe at $1.5 \mathrm{~cm}$ [shoulder of the ion probe current, Fig. 1(d)]. The peak luminescence is 1.9 times smaller than at $1.3 \mu \mathrm{s}$, and the optical absorption spectrum is still dominated by $\mathrm{Y}^{+}$absorption. During the second peak of the ion probe current at $2.9 \mu \mathrm{s}$, the probe is immersed in the brightest region of luminescence [Fig. 3(d) and 3(g)] which is half the peak intensity of that at $2.1 \mu \mathrm{s}$. In addition to the ion lines, ground-state neutral yttrium becomes noticeable [lines indicated by $\mathbf{o}$ in Fig. 3(j)].

At longer distances, a similar situation exists at lower pressures. Figure 4 shows a comparison of ion probe current, optical emission intensity (of $\mathrm{Y}^{*}$ and $\mathrm{Y}^{+*}$ ), and optical 
absorbance ( $\mathrm{f} \mathrm{Y}$ and $\mathrm{Y}^{+}$) time dependences at $\mathrm{d}=2.5 \mathrm{~cm}$ following $\mathrm{KrF}$-laser irradiation of yttrium in (a) vacuum $\left(1 \times 10^{-6}\right.$ Torr) and (b) $122 \mathrm{mTorr}$ argon. In vacuum [Fig. 4(a)], groundstate neutrals and ions are found (through optical absorption) in coincidence with excited ions and neutrals (from optical emission) on the leading edge of the ion probe current. In background argon [Fig. 4(b)], excited and ground-state $\mathrm{Y}^{+}$ions dominate the first peak of the ion probe waveform, while excited and ground-state neutrals appear in the delayed, second distribution. This combined data indicates relative depopulation of neutrals (and an associated increase in ions) in the 'fast' component of the plume penetrating through the background gas.

Luminescence from different species in the ablation plume reveals notable spatial and temporal variations both in vacuum and in background gas, as shown in Figure 5, however the general features described above for yttrium are generally preserved. Vacuum and background gas (75 mTorr oxygen) images of emission from excited $\mathrm{Ba}^{*}(553.5 \mathrm{~nm} \pm 5 \mathrm{~nm})$ and YO* (598 $\mathrm{nm} \pm 5 \mathrm{~nm}$ ) at $\Delta \mathrm{t}=2.2 \pm 0.05 \mu \mathrm{s}$ following $1.5 \mathrm{~J} / \mathrm{cm}^{2}$ ablation of YBCO are presented in Fig. 5 (b) and 5(c) and Figs. 5(e)-5(f), respectively. In each case, the background image contains, in addition to the obvious bright 'shock' region, two additional regions of luminescence: (1) a shielded, unaffected region close to the target which appears nearly equal in intensity and shape to that in vacuum, and (2) a region extending to long distances (fast component) ahead of the bright, slowing 'shock' region. Subtracting the vacuum intensities[(b) and (e)] from the background images [(c) and (f)] permits the region of strong interaction to be illustrated [(d) and (f)].

\section{CONCLUSIONS}

In summary, simultaneous application of the four diagnostic techniques confirms that a component of target material is transmitted through low-pressure background gases with little or no delay compared to vacuum. Weak optical emission from this 'fast' component originates principally from yttrium ions. Ground state and excited neutrals, which are present during the 'fast' vacuum distribution, appear delayed (or depopulated) at similar times in background gases and are correlated principally with the bright luminescence at the slowed, second peak of the ion probe signal.

F-II.8 Geohegan, et.al. p. 6 
The data are consistent with scattering over extended distances in which ions and atoms in the ablation plume undergo collisions with mean-free-paths $\sim 1 \mathrm{~cm}$ at $200 \mathrm{~m}$ Torr. Plume material is retarded and lost from the 'fast' vacuum velocity distribution due to collisional momentum transfer with the background gas (as well as other retarded plume material). This slowed material becomes observable as a distinct, second distribution only for a limited range of distances and pressures.

Alternate explanations have been proposed, including ion-ion interactions with pre-ionized background gas atoms near the target surface, ${ }^{11-13}$ however the imaging data implies interactions continuing at long distances from the target surface as evidenced by the plume luminescence. The luminescence represents populations of short-lived excited states (radiative lifetimes $\sim 10$ ns which can be populated by a variety of collisional processes, including threebody recombination of ions with electrons, electron-impact excitation of ground and low-lying states of neutrals and ions, and collisional or radiative deactivation of Rydberg states. The luminescence intensity does not necessarily correspond to the overall plume density, but is determined by velocity- or temperature-dependent rate constants and their effects on the population kinetics of luminescent levels. The enhanced luminescence in the slowed 'shock region' is explained generally by favorable population kinetics there. The depletion of groundand excited-state neutrals observed in the 'fast' distribution is more likely the result of conversion to neutral Rydberg states or ions than a preferential slowing of neutrals. A detailed experimental description of the plume luminescence and implications for plume temperatures and density-redistributions, as well as computer simulation of the above effects, will be presented elsewhere.

\section{ACKNOWLEDGMENTS}

The authors gratefully acknowledge Cambridge Research and Instrumentation, Inc. for the use of the tunable liquid crystal filter. In addition, many helpful discussions with C.-L. Liu, J. N. Leboeuf, K.-R. Chen, J. Donato, R. F. Wood, D. P. Norton, and D. H. Lowndes are appreciated. This work was supported by the Division of Materials Sciences, U.S. Department of Energy under contract DE-AC05-84OR21400 with Martin Marietta Energy Systems, Inc.

\section{F-II.8 Geohegan, et.al. p. 7}




\section{Figure Cantions}

Figure 1: Ion probe current waveforms measured along the normal to various targets at the indicated delays following 248-nm KrF-laser ablation in vacuum and background gas: (a)

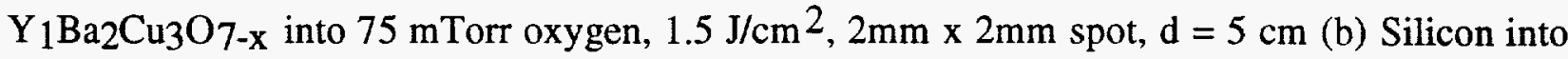
helium, $2.8 \mathrm{~J} / \mathrm{cm}^{2}, \mathrm{~d}=5 \mathrm{~cm}$, actual voltages (c) Silicon into helium (200 mTorr) and argon (80 mTorr), $2.8 \mathrm{~J} / \mathrm{cm}^{2}, 3.2 \times 2.0 \mathrm{~mm}$ spot, $\mathrm{d}=5 \mathrm{~cm}$, voltages scaled by 25 and 100 , resp. to illustrate the relative timing between vacuum and 'fast' component arrival times (d) Yttrium into 200 mTorr argon, $0.8 \mathrm{~J} / \mathrm{cm}^{2}, 2.0 \mathrm{~cm}$ line focus, $\mathrm{d}=1.5 \mathrm{~cm}$. The bimodal $200 \mathrm{mT}$ orr waveform is plotted in correct magnitude relative to the vacuum pulse and replotted scaled by a factor of 10 . The peak of the vacuum pulse corresponds to a current flux of $25 \mathrm{~A} / \mathrm{cm}^{2}$. Imaging and optical absorption spectroscopy were performed at the three times indicated in 200 mTorr argon.

Figure 2: Gated ICCD photographs of the total visible optical plasma emission during $\Delta t=1.00$ $-1.05 \mu \mathrm{s}$ following $0.8 \mathrm{~J} / \mathrm{cm}^{2} \mathrm{KrF}$-laser irradiation of yttrium metal into (a) $1 \times 10^{-6}$ Torr and (b) $200 \mathrm{~m}$ Torr argon. The peak of the grayscale palette is normalized to 7100 and 75000 counts, respectively. (c) Schematic indicating the side-on view of the yttrium target, mm-scale, and wire-ion probe position at $\mathrm{d}=1.5 \mathrm{~cm}$. (d),(e) The same photographs are replotted with a 10 grayscale palette and equal normalization to 75000 counts for direct comparison. A line-profile of the emission intensity from the irradiated spot along the target normal (f) shows the correct relative scaling between the vacuum and background images. The inset in (f) more clearly shows the same data replotted on a $0-8000$ count scale to illustrate the small, but resolvable emission in $200 \mathrm{~m}$ Torr argon out to and beyond $1.5 \mathrm{~cm}$.

Figure 3: Imaging and optical absorption spectra at the first peak, shoulder, and second peak of the ion probe waveform of Fig. 1(d) for yttrium ablation into $200 \mathrm{mTorr}$ argon. (a) Schematic of the side-on view of the $\mathrm{Y}$-target and focusing sheet of $\mathrm{KrF}$-irradiation, wire ion probe at $\mathrm{d}=1.5$ $\mathrm{cm}$, and position of Xe-lamp beam (propagating into the page) used for optical absorption spectroscopy just above the wire probe tip. (b)-(g) Gated ICCD photographs of the total visible 
optical plasma emission, $50 \mathrm{~ns}$ gates: (b) -(d) utilize the grayscale palette and are independently normalized to $10,000,20,000$, and 38,000 counts, respectively, while (e) - $(\mathrm{g})$ utilize the $10-$ grayscale palette and are all normalized to 38,000 counts. (h)-(j) Time-resolved optical absorption spectra $(\sim 2 \mathrm{~cm}$ optical path length, $d=1.5 \mathrm{~cm}, 100$-spectra averaged with $200 \mathrm{~ns}$ gates centered at the imaged times) in $200 \mathrm{mTorr}$ argon show that ground-state $\mathrm{Y}^{+}$ion lines (indicated by + ) dominate absorption at (c) $1.3 \mu \mathrm{s}$ and (b) $2.1 \mu \mathrm{s}$ (first peak, shoulder of ion probe signal): $358.45,360.07,360.19,361.10,362.87,363.31 \mathrm{~nm}$. Y neutral absorption becomes (indic. by $\mathbf{o}$ ) noticeable in the second peak of the ion probe signal as indicated in $(\mathrm{k}) 2.9$ $\mu$, at $359.29,362.09 \mathrm{~nm}$.

Figure 4: Normalized ion probe current (IP), luminescence (Lum.) intensities of $Y^{*}(410.2 \mathrm{~nm})$ and $\mathrm{Y}^{+*}(363.3 \mathrm{~nm})$, and optical absorbances (Abs.) of $\mathrm{Y}(362.1 \mathrm{~nm})$ and $\mathrm{Y}^{+}(363.3 \mathrm{~nm})$ at $\mathrm{d}=2.5$ $\mathrm{cm}$ following $0.8 \mathrm{~J} / \mathrm{cm}^{2} \mathrm{KrF}$-laser irradiation of yttrium in (a) vacuum $\left(1 \times 10^{-6} \mathrm{Torr}\right)$ and (b) 122 mTorr argon. Scaling factors: (a) Lum: $\mathrm{Y}^{*}(/ 3), \mathrm{Y}^{+*}(\times 1)$, abs: $\mathrm{Y}(\times 70), \mathrm{Y}^{+}(\times 94)$, IP (mV/81), (b) lum: $\mathrm{Y}^{*}(/ 23), \mathrm{Y}^{+*}(/ 12.5), \mathrm{Abs}: \mathrm{Y}(\times 100), \mathrm{Y}^{+}(\times 70)$, IP (mV/81).

Figure 5: Species-resolved imaging of $\mathrm{Ba}^{*}(553.5 \mathrm{~nm} \pm 5 \mathrm{~nm})$ and $\mathrm{YO} *(598 \mathrm{~nm} \pm 5 \mathrm{~nm})$ emission at $\Delta \mathrm{t}=2.2 \pm 0.05 \mu$ s following $1.5 \mathrm{~J} / \mathrm{cm}^{2}$ ablation of $\mathrm{YBCO}$ into (a), (e) vacuum and (c),(f) 75 mTorr oxygen. (d),(g) represent the difference between background and vacuum emission intensities, i.e. $(\mathrm{d})=(\mathrm{c})-(\mathrm{b}),(\mathrm{g})=(\mathrm{f})-(\mathrm{e})$.

F-II.8 Geohegan, et.al. $\quad$ p. 9 


\section{References}

1. D. B. Geohegan, p. 28 in Laser Ablation: Mechanisms and Applications, ed. by J. C. Miller and R. F. Haglund, Springer-Verlag, Heidelberg, (1991).

2. D. B. Geohegan, Ch. 5. in Pulsed Laser Deposition of Thin Films, D. B. Chrisey and G. K. Hubler (eds.), Wiley, New York (1994) and references cited therein.

3. Roger Kelly and Antonio Miotello, (Ibid).Ch. 3.

4. D. B. Geohegan, Appl. Phys. Lett. 60, 2732 (1992).

5. D.B. Geohegan and A. A. Puretzky, accepted by Appl. Phys. Lett. . (1995).

6. D. B. Geohegan,Thin Solid Films . 220, 138 (1992).

7. D. B. Geohegan, p. 73 in Laser Ablation of Electronic Materials: Basic Mechanisms and Applications, ed. by E. Fogarassy and S. Lazare, North Holland (1992).

8. J. C. S. Kools, J. Appl. Phys. 74, 6401 (1993).

9. D. B. Geohegan, pp. 165-185 in Excimer Lasers, NATO ASI Series E: Applied Sciences Vol. 265, L. D. Laude (ed.). Kluwer, Netherlands (1994).

10. D. B. Geohegan and D. N. Mashburn, Appl. Phys. Lett. 55, 2345 (1989).

11. R. R. Goforth and David W. Koopman, Phys. Fluids 17, 698 (1974).

12. David W. Koopman and R. R. Goforth, Phys. Fluids 17, 1560 (1974).

13. David W. Koopman, Phys. Fluids. 15, 1959 (1972).

F-II.8 Geohegan, et.al. p. 10 

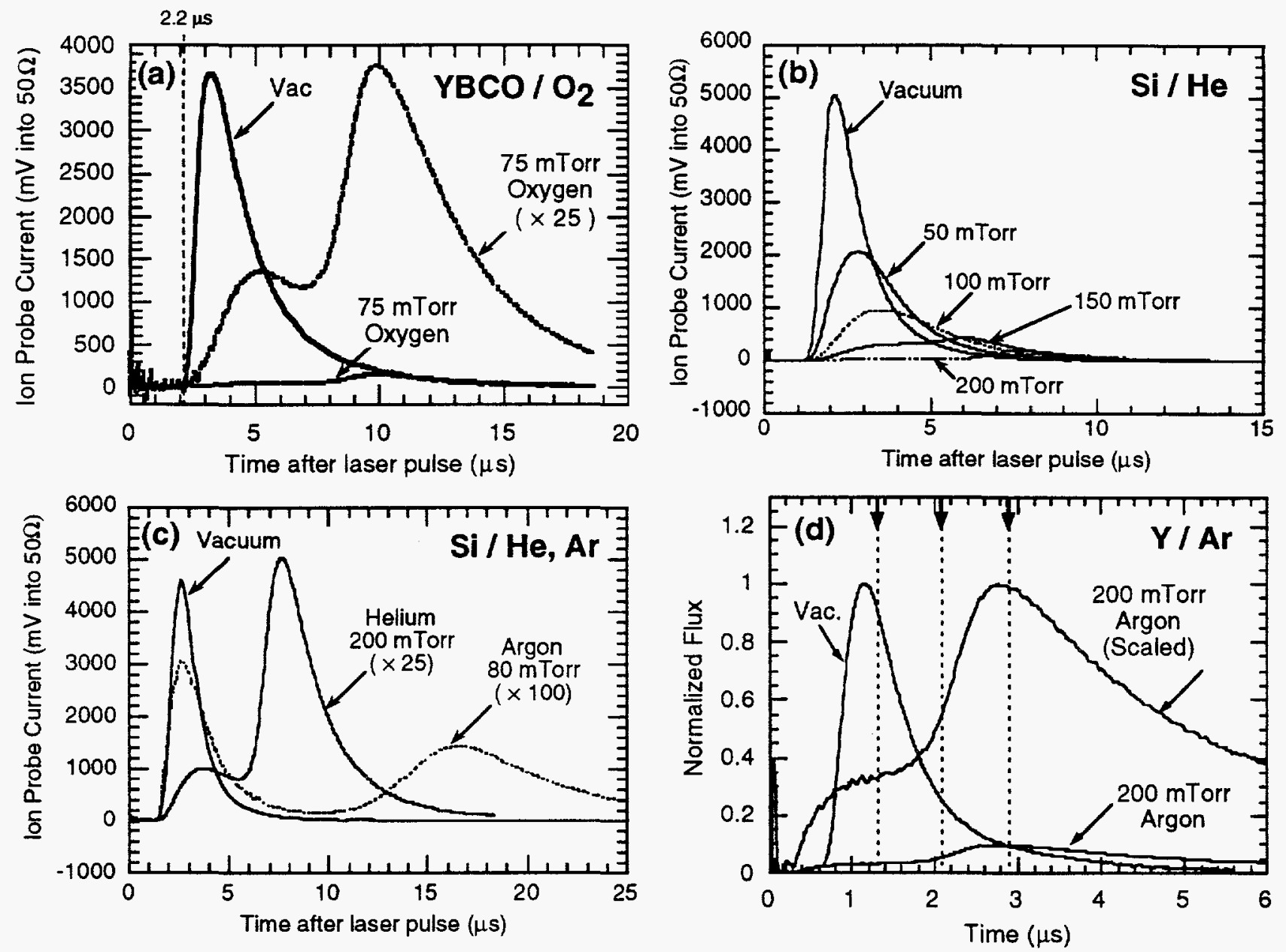

Geohegan,...

EMRS/COLA FII.8

Figure 1 

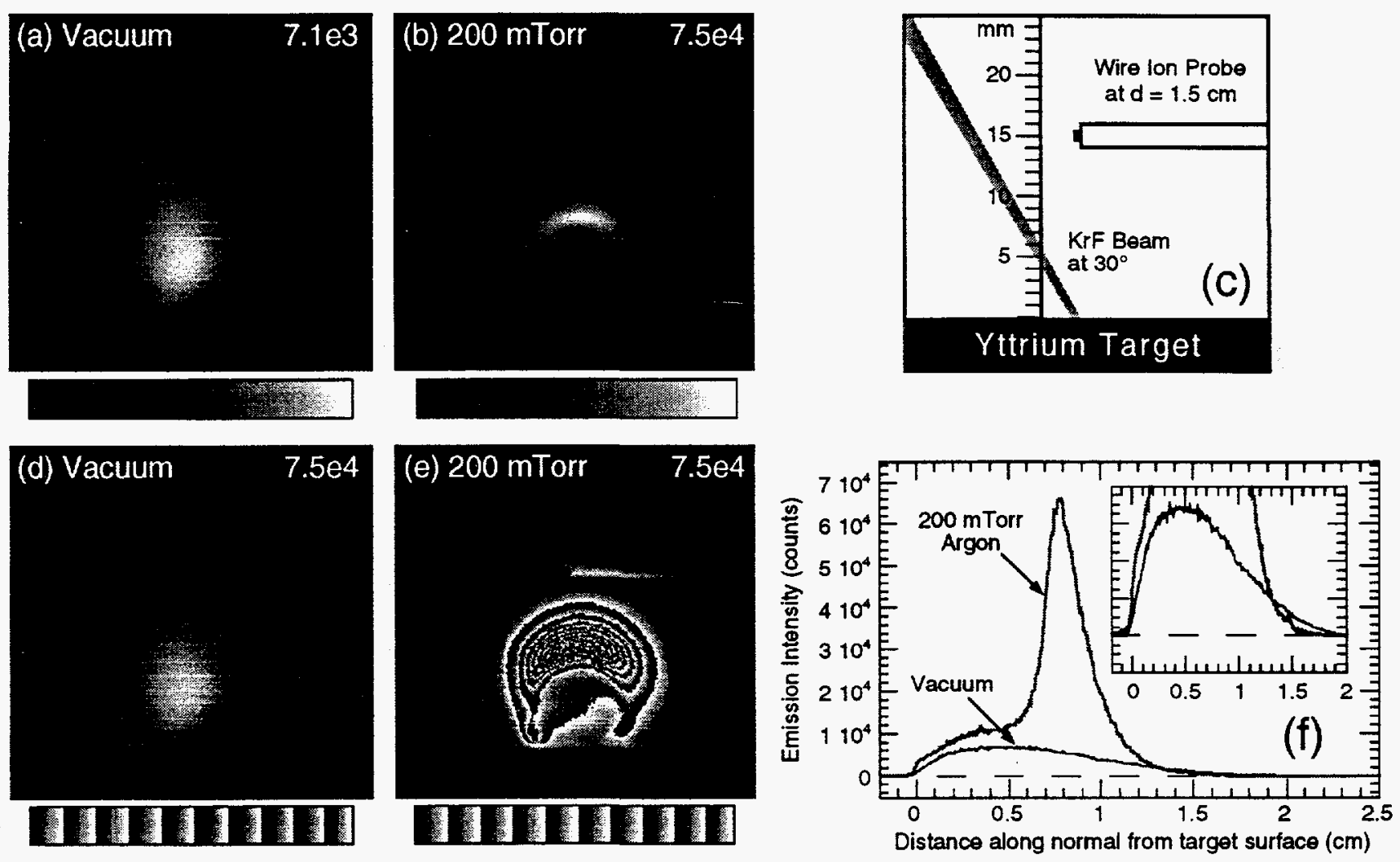

Geohegan,...

EMRS/COLA FII.8

Figure 2 


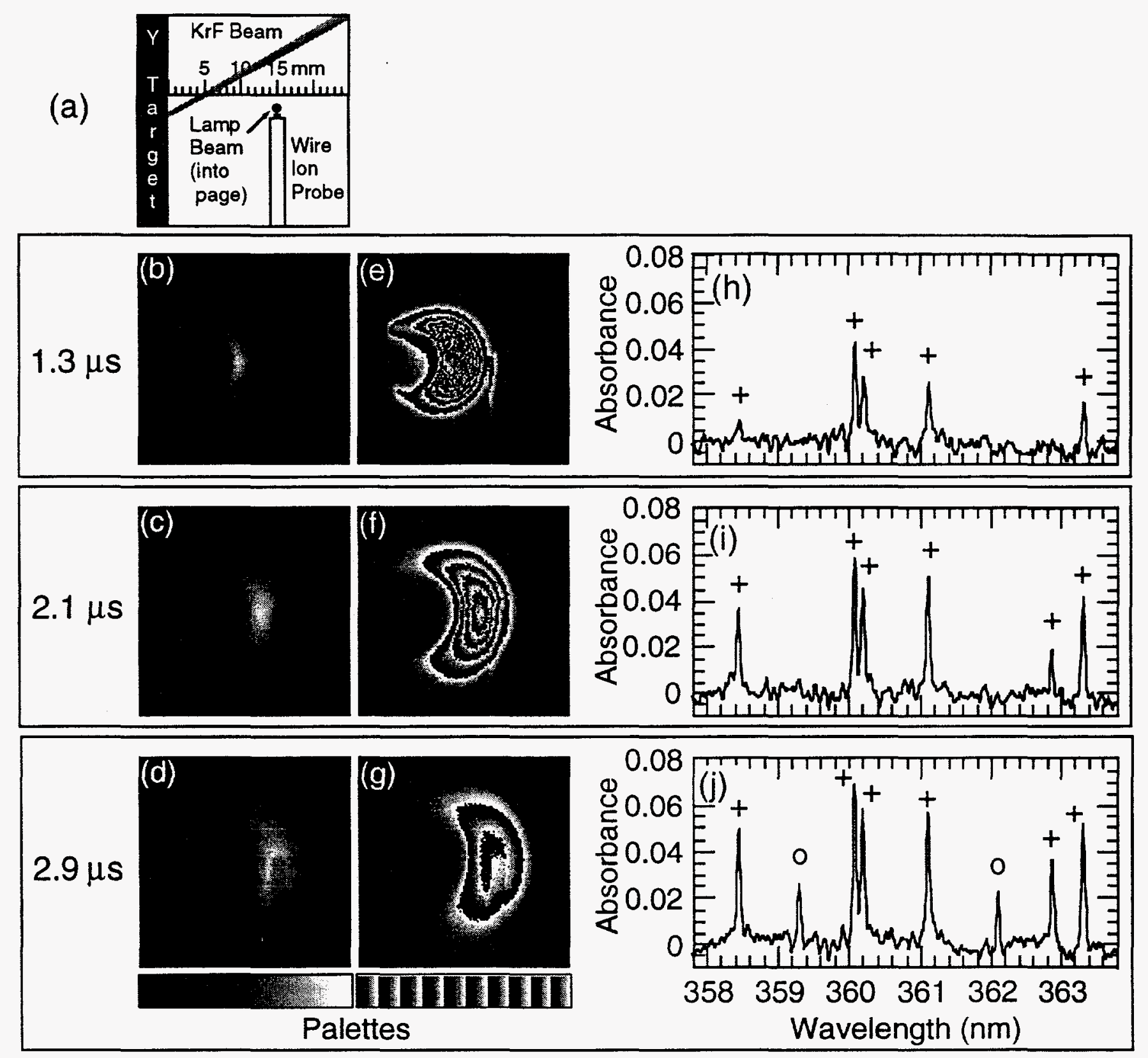

Geohegan,...

EMRS/COLA FII.8

Figure 3 

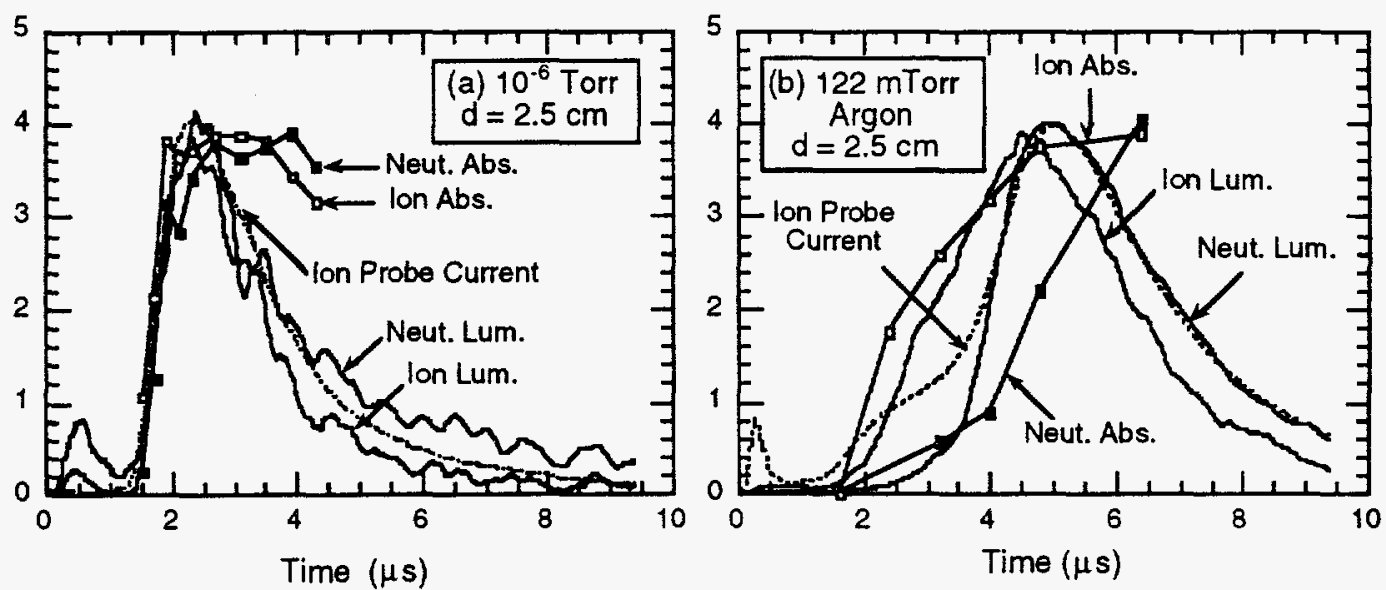

Geohegan,...

EMRS/COLA FII.8

Figure 4 


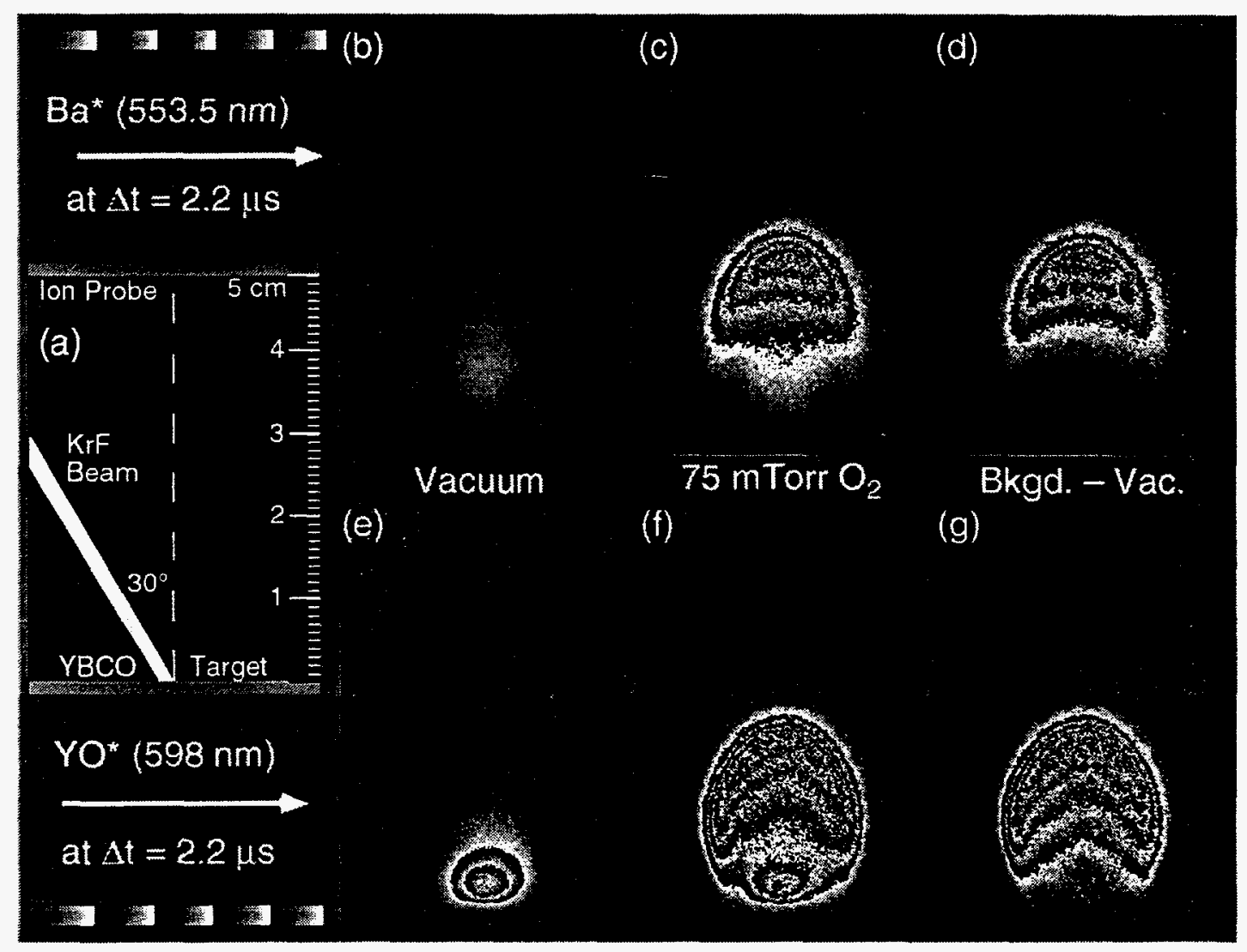

Geohegan

EMRS/COLA FII.8

Figure 5 